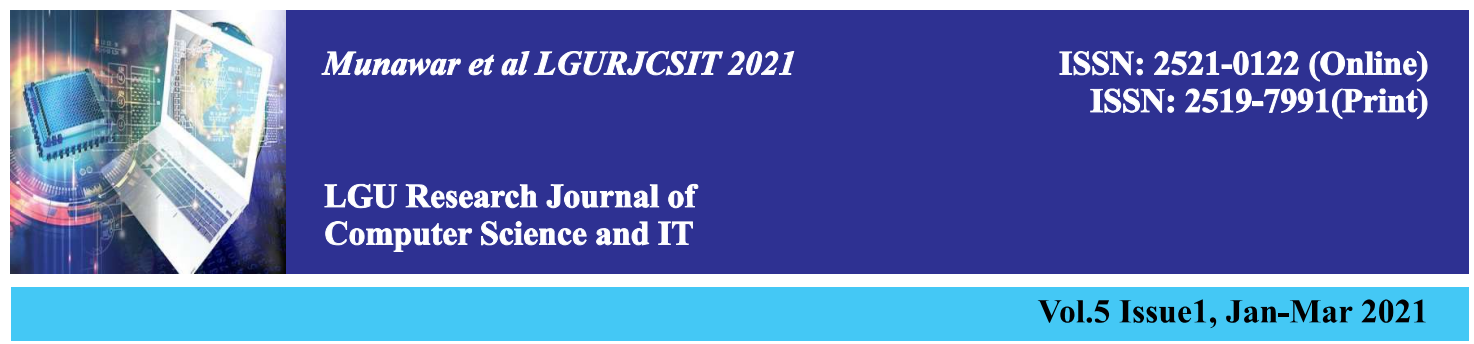

\title{
Cloud Computing: Needs Enabling Data Mining and Business Intelligent Applications
}

\author{
Qurat ul ain ${ }^{1}$, Adnan Khalid ${ }^{2}$, Aziz Guergachi ${ }^{3}$, Muhammad Shahbaz ${ }^{4}$, Awais Qasim ${ }^{2}$, Adeel Munawar $^{1}$ \\ ${ }_{1,2}$ Department of Computer Science, GC University, Lahore, Pakistan \\ ${ }^{3}$ Ted Roger's School of Management, Ryerson University Toronto, Canada \\ ${ }^{4}$ Department of Computer Science, UET, Lahore, Pakistan \\ Adeelmunawar43@gmail.com
}

\begin{abstract}
As a new computational paradigm, cloud computing is attracting a lot of interest from researchers in the field of the business community and information technology sector and it can integrate with several heterogeneous resources makes it distinguished and unique to fulfill the demands of different types of users. The rapid increase in data volume and fixed access to online resources, which is related to all departments need to mine data for the discovery of knowledge. Its principal peculiarities incorporate a versatile asset design and along this line a suitable system for tending to be comprehended in an ideal mode. From the specific situations where cloud computing can be integrated, its use in business information and intelligence also conveys the highest aspirations from data mining to updates. This study gives an outline of the recent condition of the arrangement of Cloud Computing and elaborates, its implications in Business Intelligence and Data Mining. This study defines multiple layers that are expected to create such a framework in distinctive levels of deliberation, from the fundamental equipment stages to the product assets accessible to actualize the applications. At the end of this study, a few cases related to Data mining methodologies have been relocated to the Cloud Computing paradigm.
\end{abstract}

KEYWORDS: Business Intelligence (BI), MapReduce, Data Mining (DM), Hadoop, Hash function, Serviceoriented Architecture (SOA), Service Level Agreement (SLA), Cloud Computing.

\section{INTRODUCTION}

Cloud computing is an example of modern computing but it is not a desirable idea based on grid computing. There are two similar scenarios for reducing computational costs and increasing framework adaptation. There are also differences in the way work is done. In a computational grid, a wide range of jobs are performed on different machines, separated into several smaller parts, and a comparative office for the computing. Again, through cloud computing, it is planned to allow the client to deprive the various administrations of resources in the basic building design and thus it is not prohibited and from the web, facility to direct word can offer different administration. [1].

Cloud computing is a service-oriented architecture that allows developers to reduce the complexity of computing multiple messages, including application coordination, exchange management, and security arrangements while allowing multiple steps and conventions [2].

The Cloud Computing paradigm provides infrastructure facilities such as hardware, data storage servers, and networks. These administrations provide facilities to the client without obliging them to recognize the area and different points of interest in the computing structure [3]. Now with the advancement in the latest technologies Cloud computing is fruitful for Business Intelligence (BI) applications [4-5].

In every organization data is an important asset business analysts use this data for the decisionmaking process that informs the organization executives and senior managers with current trends [6]. Data Mining (DM), information visualization, and data warehouse technologies are used to store historical data that helps business analysts in the decision-making process [7].

Managing and processing a huge quantity of data from an organization for a little period require great funding. As expressed above, with these requirements another test for the exploration group turns out with the need to adjust the frameworks to a Cloud Computing construction modeling [8]. The primary point is to reduce data management cost and needs an efficient management system that answers multiple quires and make a wide search environment. Organizations require parallelizing the effort and

LGU Research Journal of Computer Science \& Information Technology 5(1) LGURJCSIT 
enabling fault tolerance in huge data storage as well as wants reduced computational time. Organizations face difficulties in the management of data and applying Data Mining (DM) and Business Intelligence (BI) applications for knowledge discovery in data. In particular, the cloud computing paradigm provides a standard infrastructure with different levels that must be considered to execute the administration of the information, an analogous execution system that answers a query with reduced time [4].

In the case of Business Intelligence (BI) cloud computing offers great benefits with Data Mining (DM) processes and cloud virtualization [5]. It is highly expensive to parallelized high computational processes, for instance, those approaches are based on transformative models [9]-[11]. MapReduce and Hadoop are the latest two programing models used in a cloud environment that provides a platform for developers to run their algorithms.

Both frameworks have been outlined with two critical limitations: in the first place, mists have expected nods within the cloud are co-spotted, i.e. inside one server farm, on the other hand, that there is generally little data transmission accessible between the topographically conveyed groups containing the information [12]. Second, these mists have accepted that individual inputs and yields to the cloud are generally little, even though the total information oversaw and transformed are vast.

Data Mining (DM) applications are needed to apply in cloud computing because of the requirement of data management and knowledge discovery from a huge volume of data in business, science, and engineering [11], [13]. Data Mining (DM) in the cloud paradigm helps in mining knowledge from unstructured or semi-structured internet resource data. In the field of business, it is much needed for organizations to centralize the management of data, and ensure reliable, secure services for their users. Companies don't need to spend funds on the hardware infrastructure to apply data mining, they can apply mining algorithms through the browser and pay for the resources that they use.

In the most recent years, numerous Data Mining (DM) algorithms move to a cloud computing model for productive results. Day by day increase in the data volume needs high computational power for mining algorithms. This study will show a few current recommendations that adjust standard processes to the cloud for building them to be highly proficient and versatile. Developing a variety of uses in genuine building issues, for example, PC vision [4], proposal frameworks [4], or Healthcare frameworks [14] demonstrate the high essentialness of this methodology.

This study organized into sections, Section 2 presents the primary ideas on Cloud Computing, comprising its structure and fundamental layers. Area 3 and 4 introduce the structural planning ways to create Business Intelligence arrangements on a new computing paradigm. The encoding models for executing Data Mining procedures inside this ideal model, along with a few cases, are demonstrated in Section 5. At long last, the fundamental closing comments are given in the last section.

\section{BASIC ASPECTS REGARDING CLOUD COMPUTING}

Cloud computing provides a variety of services on demand over the internet with dynamic and elastic resource allocation in a multitier environment. Service-Oriented Architecture (SOA) is a blend of technological architecture that support and incorporate all kind of services [4]. The computing framework provides services on demand and is organized in a way that it can be continuously provided to the client.

Cloud computing consists of pay per user business model where resources are dynamically allocated. The taxonomy of cloud computing can be classified as i) homogeneous clouds: having the same physical and virtual architecture. ii) Heterogeneous clouds: having heterogeneity in basic, virtual or physical architecture, e.g. different types of OS or different ways of execution and access of the same application. iii) Hybrid clouds: is the combination of grid and cloud computing. Redundancy, the elasticity of resources, low time of execution, high availability, efficiency, and low cost are some of the well know features of cloud computing. The characteristics of cloud computing architecture increase the scalability and reliability, where an application running on its response in real-time instead of high resource demand, a huge quantity of customers, and an increasing volume of data. The main characteristics of Cloud computing are its loose coupling, high interoperatively, and the facility of interfaces that separate the services from the implementation and the platform. Cloud computing comprises servicesoriented layers such as Infrastructure-as-a-service (IaaS), Platform-as-a-service, and software-as-aservice (SaaS) that may have a different organizational structure.

\subsection{Infrastructure-as-a-Service (IaaS) Bottom Layer of Cloud computing}

IaaS is comprised of hardware services such as storage, computation power, server, and network with the facility of intermitted software. Client access computational resources on rent as a replacement for buying and installing their hardware and data centers. IaaS is billed for these services based on their actual usage. It is beneficial for the client, who uses what he needs and pay according to the usage. The dynamic resource allocation allows the client to enhance their resource demand according to low or high workload.

LGU Research Journal of Computer Science \& Information Technology 5(1) LGURJCSIT 
IaaS offers a variety of large infrastructure as a service to scientific researchers and technicians who need high computation power to run their tests and analysis of data. It also facilitates with the data storage store huge volume of data client can access any time when they need.

\subsection{Platform as a Service (PaaS) Middle Layer of Cloud Computing}

Platform as a Service (PaaS) is an integrated set of software with all necessary stuff that a developer needs during application development and execution. The developer does not directly access the infrastructure, the PaaS service provider offer tools that a designer might need for IaaS service [15]. PaaS is a set of independent tools that allow the developer to develop components of the application as well as the application themselves. It reduces the development cost developer no need to buy their software tool kits for making a development environment.

\subsection{Software as a Service (SaaS) Upper Layer of Cloud Computing}

This layer offers software as a service. SaaS providers offer software programs to millions of users and it was the first implantation of cloud services. The client gets benefits and reduces the cost, needs not to worry about the management of software. The third-party is responsible for updating, installing, maintaining, patching, and licensing the software.

\section{CHALLENGES AND LIMITATIONS IN CLOUD COMPUTING}

Clouding computing is a highly adopted technology in the last few years due to its characteristics Such as on-demand service, elasticity, a huge pool of resources, and a pay-per-use business model. However, it provides a variety of services but there are some limitations and challenges that need to be tackle.

3.1. Security and privacy: Data is a vital asset in any organization and plays an essential role. Data Security and privacy is the most wanted need for an organization since in cloud computing data can be distributed on multiple hardware servers managed by the third-party customer who has no control over the data. Security needs require computation of encoded data and a physically secure environment for data servers. The service provider needs to increase user confidence levels.

3.2. Service Availability and recovery: Service providers must ensure 24 hours of services with the guarantee and use efficient systems and resolve bandwidth problems.

3.3. Loss of governance: The cloud providers have overall control in services which is also a security gap that may lead to confidentiality and integrity problems.

3.4. Resource Scalability: Cloud computing technology attracts a huge number of clients, changing demand of users requires an efficient resources scheduler and monitoring system. It is necessary to reduce energy consumption.

\section{BUSINESS INTELLIGENCE (BI) PROCESSES IN CLOUD COMPUTING}

The business intelligence system needs to analyze the huge amounts of data in a very short period usually it is not more than a few minutes. Research shows enterprises are the owner of large volumes of data and it is essential to get the knowledge to predict future trends. This bulk volume of data may consequence of diverse applications, for example, forecast of advance concessions and credit strategies to customers because of dangers, characterization of a client according to their market demand, item proposals, and mining of examples from business exchanges between others.

In the rest of this area, first, present structural planning to create BI arrangements on a Cloud Computing stage. At that point, we will push the decency on the utilization of Cloud Computing concerning other comparable innovations.

\subsection{Cloud Computing Environment for Business Intelligence (BI):}

It is an essential business community to predict future trends that are hidden in a large amount of data. In [4] researchers reconsidered the essential model and procedure for investigating structured and semi-structured in a business warehouse. Cloudbased data management architecture for organizations is consists of four layers however three are the most common layer in cloud computing architecture [5]. The first three levels are regular for both methodologies, while the previous one is particularly outlined for information warehousing. The portrayal of these segments is counted underneath:

$>$ The First level takes after the structure presented in the above section, that is, it incorporates a system construction modeling with numerous approximately coupled PC nodes for giving decent scalability and an adaptation to the internal failure plan. As recommended, the framework must consider an element/ versatile plan such as the execution, expense as well as energy utilization of each node machines is overseen at run-time.

The second level is dedicated to the storage of parallel data, based on the storage and recovery of key sets (instead of the social model in light of outside key/essential key connections). A few samples of frameworks that are streamlined for this design are Google BigTable [16] or Sector [7]. 


\begin{abstract}
$>$ This level is the implementation environment. Because of the expansive number of nodes, Distributed computing is particularly pertinent for distributed computing assignments taking a shot at rudimentary operations. The most known case of a distributed computing execution environment is most likely Google MapReduce [17] and its open-source variant Hadoop [18],[19]. However, different projects can be found as doable options [20], [21]. All these situations go for giving flexibility by permitting to arrange assets as indicated by the application, taking care of blunders straightforwardly, and guaranteeing scalability.
\end{abstract}

$>$ The final level is the high-level querying language, which is situated towards OLAP. This level implicates as an interface to the client and it gives straightforwardness to alternate levels of the building design. A few query languages have been proposed like Map-Reduce-Merge [17], [22].

\subsection{Data Mining (DM) and Business Intelligence (BI) in Cloud Computing}

Generally, large-scale organizations have a huge volume of data, which essentials to be processed in a small-time, at a fast speed. For this purpose, grid computing is considered the best solution to reduce the cost while enhancing the flexibility and overall power of the system. The similitudes with Cloud Computing are apparent since both have the same characteristics such as approximately coupled, heterogeneous, and geologically scattered nodes. On the other hand, the fundamental distinction is the ways errands are processed in every particular setting. In a computational grid, one expansive occupation is partitioned into numerous little divides and executed on different machines, offering a comparable office for computing force. Then again, the computing cloud is proposed to permit the client to get different administrations without putting resources into the basic structural planning and accordingly is not all that prohibitive and can offer a wide range of administrations, from web facilitating, directly managed by a third party [4].

Also, the preferences of this new computational paradigm concerning other contending innovations are rich. First and foremost, Cloud application suppliers endeavor to give the same or better administration and execution as though the product projects were introduced provincially on end-client PCs, so the clients don't have to burn through cash purchasing a complete equipment hardware infrastructure to utilized, i.e. a basic portable gadget is sufficient to run the projects on the Cloud [4], [23].

Another advantage of, this kind of model for the data storage and the computing plans permits organizations to access their applications up and running quicker, with a lesser need of support from the IT office since it naturally deals with the business request by relegating pretty much IT assets (servers, stockpiling and/or systems administration) contingent upon the computational load progressively [24]. At last, this natural versatility of this framework makes the charging of the infrastructure to be carried out as indicated by the previous certainty.

\section{DATA MINING (DM) WITHIN THE CLOUD COMPUTING PARADIGM}

This part of the study focusing on indicating the guaranteeing future prediction for the Distributed computing paradigm in regards to the execution of DM algorithms in place to manage vast data storage for which it has been restrictively extravagant until this minute.

The ideas behind all the recommendations in this study consistently disperse the completeness of data across all nodes in the cloud and also exchange a small amount of information thus making applications exceptionally adaptable and efficient. It would be prudent, however, to maintain consistency and data protection [25].

It is important to change the data put away into the multidimensional array to "Pig data" [26] to have the capacity to do online investigation process by utilizing the MapReduce/Hadoop technology or related methodologies, (for example, Segment/Sphere [26]), likewise decreasing the stockpiling expenses [7]. In this programming model, clients determine the reckoning regarding a guide and a lessened capacity, and the basic runtime framework naturally parallelizes the processing crosswise over huge bunches of machines, machine disappointments, and timetables between machine correspondence to make productive utilization of the disks, network.

In MapReduce programing model Map, composed by the client, takes data as input and generates intermediate value pairs [27]. The MapReduce library bunches together all transitional qualities related to the same moderate key I and forward to the diminished capacity.

Then Reduce function takes an intermediate Key I and its related set of values. It blends these qualities to structure a conceivably littler arrangement of qualities [19]. The middle qualities are supplied to the client's decrease capacity through an iterator. This permits us to handle arrangements of qualities that are so vast it couldn't be possible to fit in memory.

In MapReduce, Map function summons is divided crosswise over different machines by mechanically dividing the data into an arrangement of $M$ parts. Data divided into splits are processed parallel by all machines in the cluster [22]. In this programming model, the Reduce function divides the intermediate key into $\mathrm{r}$ parts with the help of partitioning hash modulus $\mathrm{R}$ function. The number of partitions $(\mathrm{R})$ 
and the parceling capacity are indicated by the client (see figure 1)

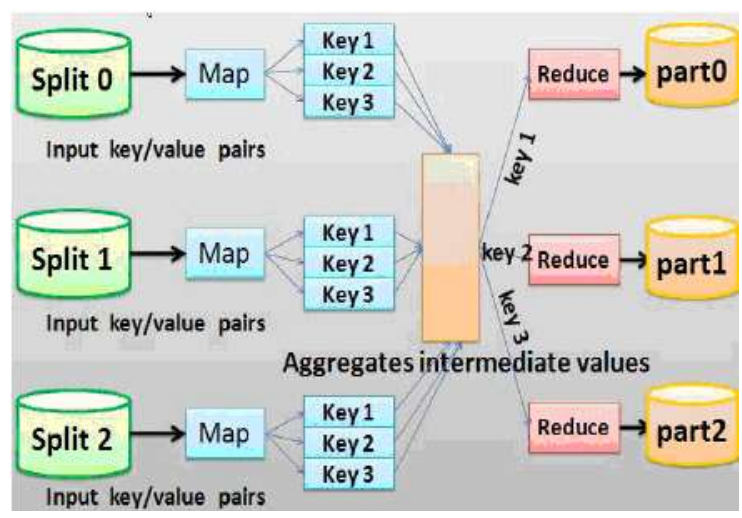

Figure 1: Map-Reduce architecture [27]

To sum things up, these frameworks are arranged to disseminate the data groups besides the cloud and to disperse the processing among the bunches, i.e. as opposed to moving all data among the machines, they characterize mapping functions to make go-between tuples of $<$ key, value $>$ and with the help of the reduced functions lesser the processing time. As a sample, in a system that went for numbering the number of events of an individual word within a vast gathering of reports, the map function will emanate every expression in addition to a related check of events though the diminished capacity totals together all checks radiated for a specific word.

Even though it is a generally new structure, numerous DM algorithms have been present effectively actualized after the rules of this programming model. Case in point, in [16]the creators display an arrangement model which tries to discover middle of the road model in the middle of Bayes and K-closest neighbor procedures by utilizing a positive sort of subtree express to every group which is gotten by bunching on preparing set by applying a base spreading over tree MapReduce execution, and after that perform the characterization utilizing thought like KNN [25].

An alternate approach that is produced utilizing the MapReduce model goes for tending to the dynamic successive example mining issue [22], which inherently experiences the scalability issue. Two Map/Reduce occupations are planned; the hopeful computing occupation registers hopeful successive examples of all arrangements and upgrades the outline of every arrangement for the future reckoning. At that point, utilizing all competitor consecutive examples as information, the bolster collecting employment gathers the event frequencies of competitor successive examples in the present time of investment and reports successive consecutive examples to clients.
Decision Tree Algorithm presents a test investigation utilizing an arbitrary Tree Algorithm under a distributed computing environment by considering two diverse plans keeping in mind the end goal to execute the parallelization of the learning stage [11], [16]. The primary methodology was that every node creates classifiers with its local data simultaneously and all classifiers are accounted for to a focal node. At that point, the focal node will utilize all classifiers together to do expectations. In this model, each node performs a smaller part of the task that may relate one or more classifiers and send its results to the focal node.

Different works are because of diverse distributed computing situations, a Particle Group Optimization was intended for Amazon Cloud, where the hopeful arrangements are displayed by the arrangement of errand administration sets, having every molecule to gain from diverse models, in any case, to take in the other practical sets for distinctive measurements. The valuable position building strategy ensures every position was demonstrated to be plausible and this plan enormously diminishes the hunt space and improves the calculation execution.

Another researcher proposed a novel DM system with the name of FD-Mine that can productively use the cloud nodes to quickly find incessant examples in distributed computing situations with information protection saved. Through observational assessments on different re-enactment conditions, the proposed FD-Mine indicated to convey fabulous execution regarding scalability and reduce processing time. A Data Mining library is available, it is open source and the user can utilize techniques implemented in this library.

\section{CONCLUSION AND FUTURE TRENDS}

This study identifies business intelligence and data mining applications in a computing environment. In particular, we use the incredible conceptual results, data mining algorithms and business intelligence to calculate high-dimensional issues that the framework deals with standard technology.

this study initially introduces the basic features of the cloud computing structure, discussing it at a diverse level that allows us to understand its trend. We presented its advantages along with the definition of other established innovations, for example, grid computing and correspondingly how this project makes data mining applications profitable. This study examines data mining (DM) and business intelligence (BI) applications in the cloud infrastructure. In particular, a four-tier structure is recommended following the advice given in the specific article. This structure allows management nodes and correspondence systems to be minimized, parallel storage of data to spread data across the cloud, and implementation situations in which the features 
offered should be exploited made up of cloud infrastructure. Bid high queries to completely misuse parallel implementation level features. Implemented data mining algorithms in the cloud environment, and reviewed the implementation features that are primarily in the light of the MapReads project, to allow efficient use of information currently available in the cloud. This is a very delicate process.

\section{REFERENCES}

[1] Xiao, Z., \& Xiao, Y, "Security and privacy in cloud computing", IEEE communications surveys \& tutorials, 15(2), 2012.

[2] Namjoshi, J., \& Gupte, “A Service oriented architecture for cloud based travel reservation software as a service",In 2009 IEEE International Conference on Cloud Computing (pp. 147-150) 2009.

[3] Bhardwaj, S., Jain, L., \& Jain, S. "Cloud computing: A study of infrastructure as a service (IAAS)", International Journal of engineering and information Technology, 2(1), (60-63),2010.

[4] Gupta, P., \& Narang, B., "Role of text mining in business intelligence.", Gian Jyoti E-Journal, 1(2) 2012.

[5] Vossen, G. "Big data as the new enabler in business and other intelligence.", Vietnam Journal of Computer Science, 1(1), 3-14, 2014.

[6] Mishra, S.,"Financial management and forecasting using business intelligence and big data analytic tools.", International Journal of Financial Engineering, 5(02), 2018.

[7] Gupta, A., Seshasai, S., Mukherji, S., \& Ganguly, "A. Offshoring: the transition from economic drivers toward strategic global partnership and 24hour knowledge factory.", Journal of Electronic Commerce in Organizations (JECO), 5(2), 1-23, 2007.

[8] Martens, B., \& Teuteberg, F., "Risk and compliance management for cloud computing services:”, Designing a reference model. Risk, 85 , 2011.

[9] Li, Q. Q., Zhang, T., \& Yu, Y. Using cloud computing to process intensive floating car data for urban traffic surveillance. International Journal of Geographical Information Science, 25(8), 1303-1322, 2011.

[10] Wu, C., Buyya, R., \& Ramamohanarao, K., "Big data analytics $=$ machine learning + cloud computing.", arXiv preprint arXiv:1601.03115, 2016.
[11] Ouf, S., \& Nasr, M.," Business intelligence in the cloud". In 2011 IEEE 3rd International Conference on Communication Software and Networks (pp. 650-655), 2011.

[12] Verma, N., \& Singh, J., “An intelligent approach to Big Data analytics for sustainable retail environment using Apriori-MapReduce framework". Industrial Management \& Data Systems, 2017.

[13] Padhy, N., Mishra, D., \& Panigrahi, R., "The survey of data mining applications and feature scope.", arXiv preprint arXiv:1211.5723, 2012.

[14] Koh, H. C., \& Tan, G., "Data mining applications in healthcare.", Journal of healthcare information management, 19(2), 65, 2011.

[15] Rani, D., \& Ranjan, R. K., "A comparative study of SaaS, PaaS and IaaS in cloud computing.", International Journal of Advanced Research in Computer Science and Software Engineering, 4(6), 2014.

[16] Hall, K. B., Gilpin, S., \& Mann, "G. Mapreduce/bigtable for distributed optimization.", 2010.

[17] Zaharia, M., Konwinski, A., Joseph, A. D., Katz, R. H., \& Stoica, "IImproving MapReduce performance in heterogeneous environments.", In Osdi (Vol. 8, No. 4, p. 7), 2008.

[18] Zhao, J., Wang, L., Tao, J., Chen, J., Sun, W., Ranjan, R., ... \& Georgakopoulos, D., “A security framework in G-Hadoop for big data computing across distributed Cloud data centres. “,Journal of Computer and System Sciences, 80(5), 994-1007, 2014.

[19] Cassales, G. W., Charao, A. S., Kirsch-Pinheiro, M., Souveyet, C., \& Steffenel, L. A., "Improving the performance of Apache Hadoop on pervasive environments through context-aware scheduling.", Journal of Ambient Intelligence and Humanized Computing, 7(3), 333-345, 2016.

[20] Cavallo, M., Di Modica, G., Polito, C., \& Tomarchio, O., "Application profiling in hierarchical Hadoop for geo-distributed computing environments.", In 2016 IEEE symposium on computers and communication (ISCC) (pp. 555-560), 2016.

[21] Gunarathne, T., Wu, T. L., Qiu, J., \& Fox, G.,"MapReduce in the Clouds for Science.", In 2010 IEEE second international conference on 
cloud computing technology and science (pp. 565572), 2010.

[22] Ranger, C., Raghuraman, R., Penmetsa, A., Bradski, G., \& Kozyrakis, C., "Evaluating mapreduce for multi-core and multiprocessor systems.", In 2007 IEEE 13th International Symposium on High Performance Computer Architecture (pp. 13-24), 2007.

[23] Al-Absi, A. A., Kang, D. K., \& Kim, M. J. , "Enhancing Dataset Processing in Hadoop YARN Performance for Big Data Applications.", In Advanced Multimedia and Ubiquitous Engineering (pp. 9-15) ,2016.

[24] Almeer, M. H., "Cloud hadoop map reduce for remote sensing image analysis.", Journal of Emerging Trends in Computing and Information Sciences, 3(4), 637-644, 2012.
[25] Maillo, J., Triguero, I., \& Herrera, F., "A mapreduce-based k-nearest neighbor approach for big data classification.", In 2015 IEEE Trustcom/BigDataSE/ISPA (Vol. 2, pp. 167172),2015.

[26] Al-Absi, A. A., Kang, D. K., \& Kim, M. J. , "Enhancing Dataset Processing in Hadoop YARN Performance for Big Data Applications.", In Advanced Multimedia and Ubiquitous Engineering (pp. 9-15) ,2016.

[27] Khezr, S. N., \& Navimipour, N. J., "MapReduce and its applications, challenges, and architecture: a comprehensive review and directions for future research.", Journal of Grid Computing, 15(3),295$321,2017$. 
